\title{
CONVERSADORES, CRONISTAS DEL EGO Y BUFONES, REFLEXIONES ACERCA DE LOS CONTENIDOS QUE SE PUBLICAN EN TWITTER
}

\author{
What People Tweet: A Methodological Approach to Exploring Language \\ Functions in Content from Twitter
}

\section{Conversadores, cronistas do ego e bufões, reflexões acerca dos conteúdos que publicam-se no Twitter}

GONZÁLEZ COGLIANO, Mabel Valeria. Universidad Casa Grande (Ecuador) mgonzalez@casagrande.edu.ec

ZEREGA GARAYCOA, María Mercedes. Universidad Casa Grande (Ecuador) tzerega@casagrande.edu.ec

Fecha de recibido: 14 de octubre de 2014

Fecha de aceptado: 4 de marzo de 2015

\section{RESUMEN}

Esta investigación plantea la realización de una aproximación metodológica para explorar las funciones de los contenidos publicados por usuarios de la red social Twitter, considerando como fundamento conceptual el modelo de Jakobson. Una vez diseñada la herramienta de análisis de contenido, se la probó y validó con un estudio de caso. Como ejemplo de aplicación, se analizaron los contenidos publicados por los usuarios más activos de sus secciones de seguidores y siguiendo de una cuenta de usuario personal. Se analizaron 11200 tuits. A partir 
del análisis se proponen rasgos que posteriormente pueden servir para generar perfiles. Estos perfiles permitirían ilustrar en qué medida los usuarios de Twitter publican sus contenidos para informar, alardear, criticar o simplemente jugar con el lenguaje. Los resultados exploran la posibilidad de que por medio del análisis de funciones y construcción de perfiles de usuarios, se pueda reflexionar en relación a la naturaleza de la comunicación 2.0 y sus implicancias en nuestras interacciones con otros.

Palabras clave: Twitter, redes sociales, comunicación 2.0, perfiles tuiteros.

\begin{abstract}
This paper presents a methodological approach to explore the functions of the contents published by Twitter users, using as a theoretical foundation the model of Jakobson. Once the analytical tool was designed, it was validated with a case study. As an example of its applicability, the content published by the most active users in the following and follower section of a personal Twitter account were analyzed. Eleven thousand two hundred tweets were analyzed. From that analysis, some treats are proposed that could serve later to generate profiles. These profiles could illustrate, in what way Twitter users publish their content to inform, to brag, to judge or simply to play with language. The results explore the possibility of by means of function analysis and construction of users profiles, it might be plausible to reflect on the nature of 2.0 communication and its effects on our interactions with one another.
\end{abstract}

Keywords: Twitter, social network, communication 2.0, Twitter profiles.

\title{
RESUMO
}

Esta pesquisa apresenta a realização de uma aproximação metodológica para explorar as funções dos conteúdos publicados por usuários da rede social Twitter, considerando como fundamento conceitual o modelo de Jakobson. Uma vez criada a ferramenta de análise de conteúdo, foi provada e validada com um estudo de caso. Como exemplo de aplicação, analisaram-se os conteúdos publicados pelos usuários mais ativos de suas seções de seguidores e seguindo de uma conta de usuário pessoal. Analisaram-se 11200 tweets. A partir da análise propõem-se rasgos que posteriormente podem servir para gerar perfis. Estes perfis permitiriam ilustrar m que medida os usuários do Twitter publicam seus conteúdos para informar, alardear, criticar ou simplesmente jogar com a linguagem. Os resultados exploram a possibilidade de que por meio da análise de funções e construção de perfis de usuários, possa-se reflexionar em relação à natureza da comunicação 2,0 e suas implicações em nossas interações com outros.

Palavras-chave: Twitter, redes sociais, comunicação 2.0, perfis twitteiros. 


\section{Introducción}

Twitter es uno de los sitios de microblogging con mayor penetración mundial, según el sitio Statistic Brain, esta red tiene, al 11 de julio de 2014, aproximadamente 645 millones de usuarios, de los cuales 115 millones han sido identificados como tuiteros activos. Twitter logra atraer a sus múltiples usuarios y motivarlos a publicar usando una única pregunta: "¿Qué está pasando?”.

Pero ¿en qué medida los usuarios responden a esta pregunta en Twitter? ¿Hasta qué punto sus publicaciones están relacionadas a ella? Algunos investigadores han explorado las implicaciones de responder o no, en la escritura de tuits, a esta pregunta. Por ejemplo, Mischaud (2007) descubrió que el $60 \%$ de los usuarios no responde directamente a la pregunta "¿Qué estás haciendo?" e infiere de este hecho que los usuarios se han apropiado de Twitter de formas distintas a las propuesta por la propia red social. Se han realizado algunos estudios que exploran los usos que las personas hacen de Twitter y los tipos de contenidos que publican, a medida que se apropian de ella. Según Java, Finin, Song y Tseng (2007) un solo usuario podría tener múltiples usos e intenciones o incluso ocupar distintos roles a la hora de usar este servicio de microblogging. En este estudio se determinaron las siguientes intenciones de las publicaciones: rutinas diarias, conversaciones, reportar noticias y compartir información. Por otro lado, se establecieron como tipos de usuarios, según contenidos publicados: a) Los informadores, o los usuarios que postean frecuentemente información que sus seguidores pueden considerar útil. b) Los amigos, que engloban a los usuarios que se conectan principalmente con su familia, amigos y compañeros de trabajo. c) Los buscadores de información, que son los usuarios que rara vez publican, pero que siguen las publicaciones de otros usuarios.

Luego de observar en qué medida los usuarios de esta red se apropian de ella, caben los siguientes cuestionamientos: ¿cuáles son las funciones predominantes de los tuits que se publican? ¿Cómo difieren los usos en relación al género (que es la única variable que se identifica en los perfiles) y al anonimato o no anonimato de los usuarios? ¿Qué metodología sería pertinente para explorar estas funciones y sus usos?

El presente trabajo es parte de un estudio² (Zerega \& González, 2012) con objetivos más amplios, que exploró las características de los procesos intersubjetivos-comunicativos que se configuran en el marco de las hipermediaciones de la red social Twitter y que tuvo como unidad de análisis el caso de una cuenta personal.

El estudio planteó tres grandes categorías de análisis: a) Subjetividad virtual. b) Procesos intersubjetivos. c) Construcción del Otro. El presente trabajo se enfoca en una parte de los resultados de la categoría b que incluyó un estudio específico alrededor de los tipos y funciones de tuits y retuits.

Los objetivos centrales de la investigación fueron a) Describir las funciones predominantes en los tuits publicados. b) Identificar las diferencias en las funciones de los tuits según el género del usuario. c) Identificar si el anonimato de la cuenta influye en el tipo de funciones utilizadas. d) Diseñar una aproximación metodológica para el análisis de las funciones de las comunicaciones en redes sociales.

1 El estudio al que se hace referencia aquí es anterior al cambio de pregunta realizado por Twitter en 2009.

2 El proyecto "Análisis de las características de los procesos intersubjetivos comunicativos que se configuran en el marco de las hipermediaciones de las redes social Twitter" fue propuesto por las autoras en 2012 en la Convocatoria interna de la Universidad Casa Grande de Guayaquil. 
El propósito de este trabajo es proponer una metodología de análisis de las funciones de los contenidos publicados por usuarios personales y, a través de una categorización de las funciones del lenguaje que emergen, tomando como base la propuesta de Jakobson, proponer rasgos que posteriormente sirvan para generar perfiles de usuarios. Estos perfiles permitirían ilustrar en qué medida los usuarios de Twitter publican sus contenidos para informar, alardear, criticar o simplemente jugar con el lenguaje.

\section{Comunicación virtual}

Algunos autores han discutido acerca de los efectos, nuevas lógicas y dinámicas que se generarían de la intersección entre tecnología y comunicación humana. Boyd (2008) hace referencia a Meyrowitz (1985) quien identifica tres dinámicas, a propósito de esta intersección: audiencias invisibles y no necesariamente copresentes, colapso de contextos debido a la falta de límites espaciales, sociales y temporales, y la borrosidad de lo público y lo privado. Según Boyd, este colapso de contextos haría cada vez más compleja la decisión, para los usuarios de las redes, a la hora de escoger sus actuaciones, el tono de sus interacciones y de los contenidos que intercambian. Esto impactaría directamente en las funciones de los contenidos que los usuarios producen y los fines de las interacciones que se establecen.

\subsection{Hipertextos, extimidades y microcelebridades}

La llegada de la postmodernidad vino a inaugurar un clima de incertidumbre en términos generales y, en particular, en torno a nuestras identidades y procesos subjetivos. La caída de los metarrelatos de la que habla Lyotard (1987) se pone de manifiesto en esta época, en la cual en palabras de Castells: "La disolución de las identidades compartidas, que equivale a la disolución de la sociedad como sistema social significativo, muy bien pudiera ser el estado de cosas de nuestro tiempo" (1999, p. 394). Es decir, aquellos referentes comunitarios, familiares, culturales están cediendo espacio a estas otras identidades, por ejemplo las que se configuran en las redes sociales que, en palabras del sociólogo Bauman (1999), nos han acostumbrado a vivir en un mundo acuoso, sin referentes sólidos a los cuales asirnos.

Adicionalmente a estas transformaciones, también está la pérdida de lo lineal del texto, de su jerarquización. Para aquellas generaciones hijas del libro impreso, el consumo o producción de textos constituía un proceso más o menos ordenado y guiado por ordenamientos subjetivos, giros, referencias que eran conducidas por el autor, por el lector. En los hipertextos, es el mismo texto el que sugiere jerarquizaciones, saltos, enlaces consigo mismo o con otros textos.

Pierre Lévy, en su trabajo ¿Qué es lo virtual? reflexiona acerca de esta "aura semántica” que posee el hipertexto: Pero mientras recogemos el texto sobre sí mismo, confeccionando de este modo su relación interna, su vida autónoma su aura semántica, lo relacionamos también con otros textos, con otros discursos, con imágenes, con afectos, con toda la inmensa reserva fluctuante de deseos y de signos que nos hacen ser lo que somos. Aquí no es ya la construcción del texto la que está en juego, sino la construcción del yo; construcción siempre por rehacer, siempre inacabada. (Lévy, 1999, p. 36). 
El hipertexto, según Lévy, se podría convertir, en nada más y nada menos que en aquello que contribuye "a crear, recrear y reactualizar el mundo de significaciones que nos define” (Lévy, 1999, p. 37). Cuando navegamos por esos saltos caóticos del hipertexto, no solamente consumimos información, sino también reescribimos nuestras historias haciendo uso de una nueva posibilidad narrativa, que se aleja, según Vega-Chirino (2012), de la coherencia biográfica de antaño para aproximarse a una práctica fragmentada de collage.

Por otro lado, los procesos mismos de construcción de identidad y las nociones de intimidad se están viendo desafiadas estructuralmente por las lógicas virtuales y las tiranías del ciberespacio. Sibilia (2006, 2010), reflexiona acerca de estos fenómenos por medio de la noción de extimidad, como una nueva forma de construir lo íntimo, hacia afuera y diferenciándolo de la interioridad. La autora utiliza la figura de bucear o hundirse en las profundidades para referirse a la exploración de la interioridad del siglo IX y principios del XX (en los diarios íntimos y comunicación epistolar característicos de aquellas épocas) y la contrasta con estas nuevas formas de vivir lo íntimo, como formas exteriorizadas de ser y estar en el mundo "en el sentido de que estas subjetividades se construyen y se realizan en el campo de lo visible” (Sibilia et ál., 2010, p. 23). Estos cambios en relación a lo que se considera público o íntimo afectan definitivamente las formas de comunicación hipertextual en las redes y los motivos detrás de las interacciones.

La interacción en el ciberespacio, además, nos está dando la posibilidad de poner en escena identidades de diversos tipos. Abreu (2012), quien las llama "camaleónicas y polifónicas", cita a Zafra (2007), que identifica tres tipologías de identidades: a) Coincidentes, de una alta referencia a la identidad off-line. b) Imaginarias, es decir, un personaje que no guarda relación con la identidad off-line. c) Simbólicas, como una especie de máscara o invención que se considera, incluso, más representativa que la identidad off-line. Vale entonces la pena preguntarse ¿afectará el anonimato al tipo de funciones detrás de las comunicaciones en Twitter?

Construimos extimidades, identidades múltiples, nos dirigimos a todos y a nadie en particular: las formas de interactuar han cambiado para siempre. Scolari (2008) condensa todos estos cambios de comunicación en el ciberespacio en el término hipermediaciones, que describe como "una trama de procesos de intercambio, producción y consumo simbólico que engloba una gran cantidad de sujetos, medios y lenguajes interconectados tecnológicamente de manera reticular" (p. 277).

Esta comunicación virtual, que se inscribe en nuestra ya irreversible sociedad de la información, vendrá gobernada por la liquidez de sus referentes, la tiranía de sus velocidades absolutas, la incertidumbre de caminos que posibilitan los saltos, giros y enlaces de lo hipertextual y la visibilidad y performatividad como legitimadora de la existencia. En contraste a esos antiguos buceos por aguas profundas de los que habla Sibilia, nos encontramos frente a vistosas salpicaduras de aguas superficiales que nos hacemos mutuamente a la hora de interactuar con estas nuevas reglas del juego de la comunicación en tiempos de hipermediaciones.

\subsection{Funciones de la comunicación: posibles aplicaciones al hipertexto}

La noción de funciones de lenguaje ha trascendido de la mano de Jakobson, aunque la formula considerando aportes también de otros autores (Sánchez, 2000 \& Costantini, 2004) bajo la idea de que las conductas verbales tienen una finalidad (Fernández, 2010). El planteamiento principal de la teoría es un esquema que presenta la existencia de seis funciones de lenguaje: referencial, emotiva, poética, conativa o apelativa, fática y metalingüística. 
Jakobson (1960) asume que si bien dichas funciones pueden traslaparse, el sentido del discurso vendrá dado por la predominancia de alguna:

Cada uno de estos seis factores determina una función diferente del lenguaje. Aunque distingamos seis aspectos básicos del lenguaje, nos sería sin embargo difícil hallar mensajes verbales que satisficieran una única función. La diversidad no está en un monopolio por parte alguna de estas varias funciones, sino en un orden jerárquico de funciones diferente. La estructura verbal de un mensaje depende, primariamente, de la función predominante.

Este estudio tomó como referencia la definición básica de las funciones (Doucet, 2008):

1. La función emotiva hace evidente los sentimientos del emisor en relación a algo y es de carácter afectivo y subjetivo.

2. La función referencial, denotativa o cognoscitiva es de carácter informativo e incluye mensajes que tienen como objetivo describir, referir o designar objetos o fenómenos.

3. La función poética es de carácter estético y el eje central está en la forma del mensaje.

4. La función fática se refiere a mensajes que buscan establecer o prolongar el contacto con el objetivo de afirmar, mantener o verificar la naturaleza de la comunicación.

5. La función metalingüística, como su nombre lo indica, hace referencia a mensajes que desean aclarar el sentido de los códigos utilizados. Sin embargo, existen autores como Pano (2012) que plantean la ampliación de la función metalingüística y propone la existencia de una función metalingüística explícita, en la que ubica mensajes que tienen el objetivo de desambiguar, reflexionar, traducir o enseñar otra lengua y de una función metalingüística implícita en la que incluye mensajes de tipo humorístico, irónico, juegos de palabras, entre otros, que está relacionada al estilo, pero que no puede considerarse como una función poética.

6. La función conativa, conminativa o apelativa es la que busca persuadir u obtener reacciones específicas de los receptores.

El modelo de Jakobson sigue siendo de utilidad para analizar diferentes tipos de comunicaciones. Si bien a veces la realidad puede "desbordar" los modelos y el autor basó su modelo en reflexiones alrededor de la lingüística y la poética (Fernández, 2010), en este caso en particular, puede servir de base para analizar el fenómeno del hipertexto con algunas adaptaciones y otros fenómenos de carácter lingüístico-comunicacional.

Una de las críticas al modelo es la simplificación de las funciones de la comunicación, ya que sería difícil sostener que cada comunicación humana se adscribe a una sola función o indicar que las funciones no se superponen, sin embargo es posible identificar una función que predomina en relación a las demás (Costantini, 2004; Doucet, 2008).

Desde esa concepción, la pregunta que plantea la red social Twitter "QQué está pasando?" puede ser interpretada de la misma forma que la pregunta "¿Qué está pensando?" de Facebook y encontrarse ambas, dentro de la función conativa del lenguaje, ya que su objetivo central es abrir el canal para sostener un diálogo (Suarez, 2014) con una audiencia imaginada. Esta idea se sostiene en la concepción de que el fin principal de una red social es interactuar.

Este modelo plantea como elementos del modelo al emisor, el contexto, el mensaje, el contacto, el código y el destinatario. Si bien se asume que este modelo lineal podría ser limitante, especialmente en el contexto 
académico contemporáneo de los campos de la comunicación, en el cual, por ejemplo, desde la semiótica se plantea actualmente a la recepción como activa, al usuario de redes sociales como un prosumidor que no solo consume, sino que produce información (Scolari, 2008) y a la necesidad de pensar los procesos desde la recepción y las mediaciones que intervienen en esta (Eco, 1987; Barbero, 1998); podríamos definir al usuario como emisor, a la red social como contexto, al tuit como el mensaje y a otro usuario como destinatario, aunque en realidad ese destinatario no debe concebirse exclusivamente como otro usuario, sino como un listado de seguidores de perfiles muy distintos, que pueden considerarse como una audiencia imaginada y que no siempre son controlados por el usuario dueño de la cuenta, en la medida en que los tuits pueden replicarse y compartirse.

El usuario de las redes se dirige a audiencias "imaginadas" que según Boyd y Marwick (2011) son similares a las audiencias ficcionales de un escritor (Ong, 2005). Entonces los usuarios tendrían una audiencia a la cual invocan y para la cual producirían contenido, participando en una práctica performativa, que las autoras llaman de microcelebridad, en la cual la audiencia es vista como un conjunto de potenciales admiradores y la autorepresentación es cuidadosamente administrada para que sea consumida por estos fans.

En la medida que los usuarios se dirigen a estas audiencias imaginadas, también en las redes empiezan a emerger otros tipos de autoconciencia (Welsch, 2009), pues mientras se dirigen a estos públicos potencialmente amables, al mismo tiempo y de manera paradójica aparece la oportunidad de reflexionar acerca de lo que son y su relación con los otros, como "soliloquios que se gritan al éter o mensajes dentro de una botella que se lanza a mar abierto, sin ningún destinatario en particular" (p. 21).

\section{Metodología}

\subsection{El caso y la muestra de estudio}

Se utilizó como caso de estudio, la cuenta @elconejo01. La cuenta se abrió en abril de 2011, se identifica con un usuario-personaje. Aunque es un usuario-personaje, la cuenta está a nombre de Tina Zerega, una de las investigadoras. Si bien se asume que cada cuenta pone en evidencia una subjetividad particular del usuario que tiene unos criterios específicos para seleccionar cuentas qué seguir, unas maneras y estilos de comunicarse que a su vez repercuten en el tipo de usuarios que deciden seguir esa cuenta, se la escogió como ejemplo de aplicación de la metodología cuantitativa y porque permitía acercamientos cualitativos a usuarios relacionados a otras categorías de análisis del estudio original. Al momento del inicio del periodo de observación (agosto de 2012) había publicado alrededor de 17000 tuits, tenía a 213 usuarios en su sección de siguiendo y aproximadamente 800 usuarios en la sección de seguidores.

El universo estuvo constituido por todos los tuits de las secciones de seguidores y siguiendo de la cuenta @elconej001 producidos entre el 1 de agosto de 2012 a las 00:00 hasta el 15 de agosto de 2012 a las 23:59.

La muestra se estructuró a base de los usuarios de la sección seguidores y siguiendo que fueran más activos. Se definió la variable actividad calculando un promedio simple entre el número total de tuits de todos los usuarios del universo, publicados desde la apertura de sus cuentas hasta el 11 de julio a las 23:59 y se definió como usuarios con alto nivel de actividad a aquellos que tuvieran un número de tuits publicados por encima de dicho promedio. 
El promedio de tuits en el caso de los listados de la sección siguiendo fue de 8000 tuits y en el caso de la sección seguidores de 4000 a la fecha de realización del estudio.

Los tuits publicados por los usuarios que componen la muestra fueron descargados y almacenados en una matriz registrando fecha, hora y usuario que los publicó. Se estudiaron usuarios de carácter personal, dejando de lado los corporativos. Otro criterio de selección fue la localidad. Se eligieron usuarios nacionales (ecuatorianos), ya que el estudio demandaba acercamientos posteriores de tipo presencial.

Se dividió a los usuarios de la muestra en hombres, mujeres y anónimos. La operativización de estos aspectos se asumió como problemática, dado que muchos usuarios de Twitter no utilizan ni su nombre ni su foto verdadera para identificarse en la red. Se decidió definir la categoría masculino y femenino no anónimo, respectivamente, como cuentas que deben contener un nombre o foto masculina o femenina (no de personaje de la industria cultural), donde se identifique que la cuenta es manejada por un usuario de sexo masculino o femenino. Además, debe identificar su cuenta con un nombre completo (nombre, o sigla y apellido), dentro de la cuenta debe colocar también su nombre completo o sigla y apellido y mostrar una foto personal o de apariencia personal o incluir otro link de hipertexto en el que identifica su nombre completo: blog personal o colaboración en página web. Por otro lado, se definió la categoría anónimo a aquellas cuentas cuyos usuarios no publican nombres, ni apellidos, ni fotos de su rostro en la red social, ni otro link de hipertexto en el que identifica su nombre completo: blog personal o colaboración en página web. La justificación de esta clasificación es identificar si los aspectos de género o anonimato influyen en el tipo de contenidos que se publica predominantemente en Twitter.

Se clasificó primero a todos los tuiteros de la muestra, dentro de estas tres tipologías de usuarios (Mujeres, Hombres y Anónimos) para luego facilitar el llenado del campo Tipo de usuario en la ficha de análisis. En total se analizaron 24 cuentas de usuarios y un corpus textual de 11200 tuits.

Tabla 1. Tuits producidos según tipo de usuario. Se documentaron los tuits producidos por las veinticuatro cuentas analizadas durante el periodo del 1 de agosto de 2012 a las 00:00 hasta el 15 de agosto de 2012 a las 23:59

\begin{tabular}{|l|l|l|}
$\begin{array}{l}\text { No. cuentas según } \\
\text { tipo de usuario }\end{array}$ & $\begin{array}{c}\text { No. tuits analizados } \\
\text { por tipo de usuario }\end{array}$ & \multicolumn{1}{l|}{ Tipo de usuario } \\
\hline 9 & 2873 & Usuarios hombres \\
\hline 6 & 3516 & Usuarios mujeres \\
\hline 9 & 4811 & Usuarios anónimos \\
\hline $\mathbf{2 4}$ & $\mathbf{1 1 2 0 0}$ & Total \\
\hline
\end{tabular}

Fuente: elaboración propia. 


\subsection{El diseño de la técnica del análisis de contenido de las funciones}

Para identificar elementos de esos procesos intersubjetivos e interacciones, se escogió la técnica de análisis de contenido que según Bardín (2002), es "el conjunto de técnicas de análisis de las comunicaciones tendentes a obtener indicadores (cuantitativos o no) por procedimientos sistemáticos y objetivos de descripción del contenido de los mensajes permitiendo la inferencia de conocimientos relativos a las condiciones de producción/recepción (contexto social) de estos mensajes" (Bardín, 2002, p. 32). El análisis de contenido tiene dos finalidades (Bardín, 2002): a) Heurísticas, porque permite "ver" determinado fenómeno, en este caso describir las funciones de la comunicación. b) De administración de prueba, que en este caso nos permitió el diseño de la herramienta.

En este caso, se realizó un análisis de contenido de tipo descriptivo de carácter horizontal extensivo (Piñuel, 2002), a partir de un sistema de codificación de presencia y frecuencia (Abela, 2002). La unidad de registro fue el contenido del tuit completo.

Finalmente, se elaboró la ficha de análisis, basada en las seis funciones del lenguaje propuestas por Jakobson: referencial, poética, conativa, fática, emotiva y metalingüística (1948). Las definiciones de cada función sirvieron entonces, como punto de partida para hacer una primera exploración de su utilidad para clasificar tuits. Se desarrollaron categorías de carácter temático (Piñuel, 2002).

Se incluyó en la ficha los siguientes campos: Nombre del usuario, Tipo de usuario, Texto del tuit analizado, Link del tuit analizado y las funciones creadas.

Se analizaron para este propósito un total de 200 tuits de la muestra para validar la ficha y evaluar si las categorías y subcategorías planteadas eran exhaustivas, claras, mutuamente excluyentes y replicables (Abela, 2002). A partir de esa prueba, se decidió desglosar las categorías de las seis funciones de Jakobson en subcategorías cuyo nombre fuera más descriptivo y se ajustara mejor al tipo de contenidos que se publica en Twitter, el desglose se dio como se detalla a continuación:

Tabla 2. Categorías y subcategorías

\begin{tabular}{|c|c|}
\hline $\begin{array}{l}\text { Categoría } \\
\text { Función }\end{array}$ & Subcategoría \\
\hline Función referencial & $\begin{array}{ll}\text { 1. } & \text { Vigilar (denunciar en tono serio) } \\
\text { 2. } & \text { Hacer crónica urbana } \\
\text { 3. } & \text { Hacer crónica del ego } \\
\text { 4. } & \text { Compartir información } \\
\text { 5. } & \text { Auto reconocerse } \\
\text { 6. } & \text { Reconocer a otro } \\
\text { 7. } & \text { Debatir }\end{array}$ \\
\hline Función fática & 8. Conversar \\
\hline Función conativa & 9. Comercializar o vender \\
\hline Función emotiva & $\begin{array}{l}\text { 10. Expresar amor } \\
\text { 11. Expresar solidaridad social } \\
\text { 12. Expresar odio }\end{array}$ \\
\hline
\end{tabular}




\begin{tabular}{|l|l|}
$\begin{array}{l}\text { Categoría } \\
\text { Función }\end{array}$ & Subcategoría \\
\hline Función poética & $\begin{array}{l}\text { 13. Poetizar } \\
\text { 14. Filosofar }\end{array}$ \\
\hline Función metalingüística (implícita) & $\begin{array}{l}\text { 15. Jugar con el lenguaje } \\
\text { 16. Ironizar (denunciar en tono irónico) }\end{array}$ \\
\hline
\end{tabular}

Fuente: elaboración propia a partir del modelo de Jakobson.

La función referencial se subdividió considerando las reflexiones de la teoría de redes sociales en relación a la problemática de lo íntimo y lo público que se presenta en las redes. Esa subcategorización permitiría observar si los procesos subjetivos están implicados en lo público (vigilar, hacer crónica urbana, debatir) o en lo íntimo (hacer crónica del ego, auto reconocerse) bajo estas lógicas del yo-pantalla (Sibilia, 2006, 2010).

Además se agregó la categoría "No determinado", para clasificar contenidos hipertextuales (links a videos, audios, etc.) y textuales (signos de puntuación, números, monosílabos) que sin otro contexto explícito en el cuerpo del tuit, fuera imposible categorizar. Se detallan aspectos del proceso y del protocolo de sistematización para que pueda servir de referencia: las categorías creadas y una breve definición que sirvió para volverlas operativas:

1. Vigilar, denunciar: la función del tuit es de denuncia, reclamo de una situación política, social o crítica de un ámbito profesional. Los tuits pueden ser reclamos directos a una persona u organismo identificado o en general. Se incluyen tuits o retuits con esa función. El tono es serio, formal. Se propone como único nivel de subcategorización al objeto de vigilancia por parte del usuario:

1.1 Vigilar nivel local: hace referencia a una problemática de su ciudad natal.

1.2 Vigilar nivel nacional: hace referencia a una problemática de su país.

1.3 Vigilar nivel regional: hace referencia a una problemática latinoamericana.

1.4 Vigilar nivel global: hace referencia a una problemática de otros países fuera de América Latina.

1.5 Vigilar relativo a la moral: hace referencia a valores de otros, personajes públicos y/o usuarios seguidores/siguiendo a valores o creencias de otros.

1.6 Vigilar relativo al consumo: hace comentarios de productos o servicios.

La subdivisión de la función Vigilar, nos pareció relevante en la medida en que podría permitir visibilizar las nuevas territorialidades y ciudadanías globales.

2. Hacer crónica urbana: la función del tuit es documentar (visualmente o textualmente) el entorno urbano: frases, gastronomía, diseños, espacios. El tono del tuit puede ser formal-descriptivo o humorístico. Se incluyen tuits o retuits con esa función.

3. Hacer crónica del ego: la función del tuit es documentar la vida del usuario (sea de forma visual o textual, expresado con gráficos, símbolos, emoticones). Esto incluye acciones, espacios, recorridos, personas que lo acompañan, agenda. Abarca declaraciones en torno a reflexiones personales, notas mentales, gustos o deseos personales, etc. Se incluyen tuits que podrían considerarse como "catarsis personal". Esto es: comunicación de estados emocionales, prácticas sociales, consumos culturales, reflexiones personales. 
4. Compartir información: la función del tuit es compartir información de cualquier tema (textual, visual) como fotos de otros, artículos, videos. Pueden simplemente describirse o ser recomendados. Se incluyen tuits o retuits con esa función. No se incluyen los tuits de compra-venta.

5. Auto reconocerse: la función del tuit o del retuit es comunicar al timeline algo que otro dice sobre ti: felicitación, reconocimiento, etc.

6. Reconocer a otro: la función del tuit es reconocer a otro: felicitarlo por un logro, producto, texto, situación (cumpleaños, nacimiento, etc.).

7. Debatir: la función del tuit es interactuar con un(os) usuario(s) específico(s) para iniciar un debate de ideas. Los tuits categorizados en esta función deben cumplir dos condiciones, deben contener una mención y su contenido debe hacer referencia a cualquier intercambio de opinión o debate en torno a ideas sociales, políticas, económicas, opinión sobre productos, etc. No se incluyen retuits.

8. Conversar: la función del tuit es interactuar con un(os) usuario(s) específico(s) para iniciar una conversación. Los tuits categorizados en esta función deben cumplir dos condiciones, deben contener una mención y su contenido debe hacer referencia a temas de coordinación de lugares, dar información solicitada, bromas sin argumentación específica. No se incluyen retuits.

9. Comercializar o vender: la función del tuit es promocionar una marca, comprar o vender algo. Se incluyen tuits o retuits con esa función.

10. Expresar amor: la función del tuit es comunicar amor públicamente a una pareja específica o indeterminada. También se entiende por amor al amor familiar, fraterno o amistades. Se incluyen tuits "no te ama si". Se incluyen tuits o retuits con esa función.

11. Expresar solidaridad social: la función del tuit es presentar o compartir información de tipo solidario: persona extraviada, lugar de robo, necesidad de pintas de sangre, necesidad de medicamentos, etc. Se incluyen tuits o retuits con esa función.

12. Expresar odio: la función del tuit es enviar un mensaje que insulta a alguien específicamente: debe especificarse usuario o cuenta. No se incluyen retuits.

13. Poetizar: la función del tuit es crear textos líricos. Los textos pueden ser de creación personal o citas. Se incluyen tuits y retuits de esa función.

14. Filosofar: la función del tuit es reflexionar sobre temáticas generales: la vida, el amor, la muerte. Se incluyen tuits o retuits con esa función.

15. Jugar con el lenguaje: la función del tuit es principalmente mostrar ingenio. El creador del tuit crea juegos de lenguaje, bromas, chistes, etc. El tuit incluye creaciones del usuario o citas-referencias de otros usuarios. Se incluyen tuits o retuits con esa función.

16. Ironizar: la función del tuit es similar a la anterior, pero el tono es humorístico, sarcástico o irónico. Son tuits irónicos de denuncia, reclamo de una situación política, social o crítica de un ámbito profesional. Los tuits pueden ser reclamos directos a una persona u organismo identificado o en general. Son tuits que hacen reclamos al gobierno local o nacional, a un gremio/organismo y/o corporación. Se incluyen tuits o retuits con esa función.

Se propone también como único nivel de subcategorización al tipo de contenido que se hace referencia en el tuit: 
16.1 Ironizar nivel local: hace referencia a una problemática de su ciudad natal.

16.2 Ironizar nivel nacional: hace referencia a una problemática de su país.

16.3 Ironizar nivel regional: hace referencia a una problemática Latinoamericana.

16.4 Ironizar nivel global: hace referencia a una problemática de otros países fuera de América Latina.

16.5 Ironizar relativo a la moral: hace referencia a valores de otros, personajes públicos y/o seguidores/ siguiendo.

16.6 Ironizar relativo al consumo: hace comentarios de productos o servicios.

Sin embargo estas subcategorías y descripciones podrían mejorarse a partir de nuevas aplicaciones.

Aquí se presentan algunos ejemplos de tuits categorizados:

Tabla 3. Ejemplos de tuits por función. Los tuits colocados como ejemplo en esta tabla fueron tomados indistintamente de las tres tipologías de usuarios

\begin{tabular}{|c|c|}
\hline Ejemplo de tuit categorizado en esta función & Categorias \\
\hline Chuuuuchucara papa mote huevo? Birri birri jaja te acuerdas de eso? & Conversar \\
\hline $\begin{array}{l}\text { Siempre tengo hambre, pero esta vez es criminal. Tengo ganas de comerme a mi } \\
\text { coworker! }\end{array}$ & Hacer crónicas del ego \\
\hline $\begin{array}{l}\text { Si leí que estaba aquí No puedo:( la revolución ciudadana me ha dejado sin } \\
\text { asistencia domestica desde hace meses }\end{array}$ & Ironizar \\
\hline $\begin{array}{l}\text { One in } 5 \text { Hong Kong women will never find a husband, as city's gender } \\
\text { imbalance widens }\end{array}$ & Compartir información \\
\hline $\begin{array}{l}\text { Los DDHH también son utilizados para, so pretexto de protegerlos, cobrar } \\
\text { facturas pendientes con inst. del Estado \#notodossonhumanistas }\end{array}$ & Vigilar, denunciar \\
\hline $\begin{array}{l}\text { Qué porquerías que han puesto en las cajetillas de cigarrillos. Todo bien con la } \\
\text { salud, pero ¿y la dignidad? }\end{array}$ & Debatir \\
\hline ¿Eres libro? ¿Eres película? o ¿eres versátil? & Jugar con el lenguaje \\
\hline $\begin{array}{l}\text { We are confronted with the monstrosity of the totalitarian state. All are to think } \\
\text { alike. No one is to disagree. W. Churchill }\end{array}$ & Filosofar \\
\hline Soy lo máximo, no hay nada que hacer & Auto reconocerse \\
\hline $\begin{array}{l}\text { Los estudiantes de la escuela Aurora Vallejo salieron de sus aulas para saludar } \\
\text { al Jefe de Estado y pedir autógrafos }\end{array}$ & Hacer crónicas urbanas \\
\hline $\begin{array}{l}\text { Hoy arranca el festival Ciudad Mínima, ifelicitaciones y mucha suerte a } \\
\text { @HembraDragon y @adeljar! }\end{array}$ & Reconocer a otro \\
\hline Necesito ilustrador para crear personaje $2 d$. Info por DM & Comercializar \\
\hline $\begin{array}{l}\text { @MashiRafael Más antiético es hacerlo "escondido" tras una banda presidencial } \\
\text { y una sonrisa gingival. Qué patético bocón que es usted }\end{array}$ & Expresar odio \\
\hline Kevin Costner & No Determinado \\
\hline
\end{tabular}




\begin{tabular}{|l|l|}
\hline Ejemplo de tuit categorizado en esta función & Categorías \\
\hline $\begin{array}{l}\text { Ampollas de PROSTOGLANDINA E1 o el genérico PROSTIN o } \\
\text { ALPROSTADIN, para el corazón de recién nacido se necesitan de urgencia. } \\
\text { Gracias por RT }\end{array}$ & Expresar solidaridad social \\
\hline $\begin{array}{l}\text { All religions are the same. Religion is basically guilt with different holidays. } \\
\text { CathyLadman, American comedian/actress }\end{array}$ & Poetizar \\
\hline Sentir la vida a través de la sensibilidad de tu hijo chiquito & Expresar afecto \\
\hline
\end{tabular}

Fuente: elaboración propia.

\section{Resultados}

En la siguiente tabla se detalla el número de cuentas analizadas y el número de usuarios según tipo de usuario.

\subsection{Funciones de tuits según tipología de usuario}

En la tabla 4 se observan los números totales de tuits producidos y categorizados en las funciones. Al realizar la categorización de los tuits se reveló que las tres categorías con mayor producción de tuits variaban según tipologías de usuarios. Es así como, aunque Ironizar y Conversar se encuentran entre las primeras cinco posiciones en las tres tipologías, ocupan distintos lugares dependiendo del género y anonimato del usuario. Por ejemplo, la función Conversar en los usuarios mujeres ocupa el segundo lugar, mientras que en los usuarios anónimos y los usuarios hombres ocupa el primero. Por otro lado, la función Ironizar ocupa el primer lugar en producción total de tuits en las mujeres y el cuarto lugar en los usuarios anónimos y los hombres. Finalmente, la función Hacer crónicas del ego, aparece en segundo lugar para anónimos y hombres y en tercer lugar para las mujeres, y la función jugar con el lenguaje, aparece en tercera posición para los usuarios anónimos y en última con cero producción de tuits para los hombres.

Tabla 4. Tuits analizados según funciones

\begin{tabular}{|l|l|l|l|l|}
\hline Total por categoría & $\begin{array}{l}\text { Usuarios } \\
\text { anónimos }\end{array}$ & $\begin{array}{l}\text { Usuarios no } \\
\text { anónimos } \\
\text { mujeres }\end{array}$ & $\begin{array}{l}\text { Usuarios no } \\
\text { anónimos } \\
\text { hombres }\end{array}$ & Categorías \\
\hline 3396 & 1672 & 756 & 968 & Conversar \\
\hline 2589 & 1299 & 607 & 683 & Hacer crónicas del ego \\
\hline 1678 & $\mathbf{4 5 4}$ & $\mathbf{8 5 2}$ & $\mathbf{3 7 2}$ & Ironizar \\
\hline 1616 & $\mathbf{9 2 5}$ & $\mathbf{2 7 6}$ & $\mathbf{4 1 5}$ & Compartir información \\
\hline 606 & 80 & $\mathbf{4 3 6}$ & 90 & Vigilar, denunciar \\
\hline
\end{tabular}




\begin{tabular}{|c|c|c|c|c|}
\hline Total por categoria & $\begin{array}{l}\text { Usuarios } \\
\text { anónimos }\end{array}$ & $\begin{array}{l}\text { Usuarios no } \\
\text { anónimos } \\
\text { mujeres }\end{array}$ & $\begin{array}{l}\text { Usuarios no } \\
\text { anónimos } \\
\text { hombres }\end{array}$ & Categorías \\
\hline 412 & 111 & 86 & 215 & Debatir \\
\hline 324 & 159 & 165 & 0 & Jugar con el lenguaje \\
\hline 137 & 32 & 63 & 42 & Filosofar \\
\hline 127 & 0 & 102 & 25 & Auto reconocerse \\
\hline 90 & 20 & 66 & 4 & Hacer crónicas urbanas \\
\hline 71 & 12 & 40 & 19 & Reconocer a otro \\
\hline 39 & 5 & 22 & 12 & Comercializar \\
\hline 29 & 16 & 8 & 5 & Expresar odio \\
\hline 27 & 14 & 1 & 12 & No determinado \\
\hline 26 & 4 & 11 & 11 & Expresar solidaridad \\
\hline 24 & 0 & 24 & 0 & Poetizar \\
\hline 9 & 8 & 1 & 0 & Expresar amor \\
\hline 11200 & 4811 & 3516 & 2873 & Total \\
\hline
\end{tabular}

Fuente: elaboración propia.

La tabla está ordenada de mayor a menor número de tuits totales producidos por función. Los valores resaltados en negrita corresponden a las cinco funciones con mayor producción de tuits dentro de cada tipología de usuario.

La producción de tuits en la función Conversar, en las tres tipologías categorizadas, corresponde a la cuarta parte de producción total de tuits de las 24 cuentas analizadas. Se observa además que el número de tuits con contenidos que revelan intereses en lo público (vigilar, ironizar) es inferior al número de tuits relacionados a lo íntimo o el fenómeno de yo-pantalla (crónica del ego).

En la gráfica 1 se puede observar con mayor claridad de qué manera varía la producción total de tuits según la tipología del usuario que los publica. Se observa, por ejemplo, que hay funciones predominantes en una tipología y casi inexistentes en las otras, como es el caso de vigilar-denunciar que aparece con aproximadamente 400 tuits en las mujeres y menos de 100 para los usuarios anónimos y los hombres.

Es decir, las funciones predominantes de cada tipología de usuario sí presentaron variaciones. Aunque, en términos generales, fueron las usuarias las que difirieron en mayor medida, mientras que los hombres y los usuarios anónimos mantuvieron muchas similitudes entre sí, en sus funciones predominantes. 


\section{Gráfica 1. Cinco funciones con mayor producción de tuits según tipología de usuario}



Fuente: elaboración propia.

En las gráficas 2 y 3 se pueden observar las subcategorías de las funciones vigilar-denunciar e ironizar, comparadas según género de los usuarios. En ambas funciones resalta una subcategoría predominante y distinta según el género. Las mujeres produjeron más tuits dentro de la subcategoría vigilar nivel local, equivalentes al $19 \%$ de la producción total dentro de esta función y los hombres produjeron más tuits en la subcategoría Ironizar nivel nacional, equivalentes al $76 \%$ de la producción total dentro de esta función.

\section{Gráfica 2. Subcategorías dentro de la función Vigilar según género}

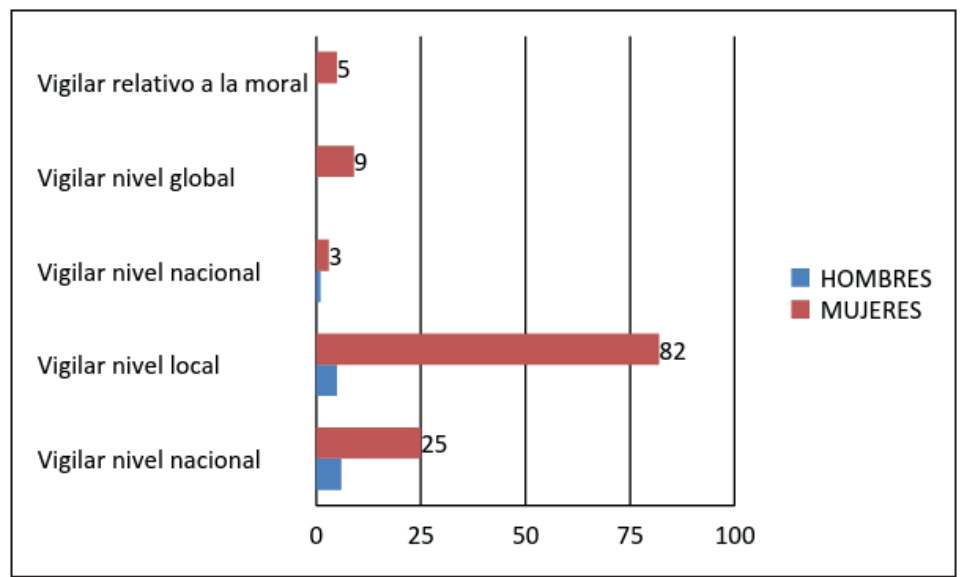

Fuente: elaboración propia. 


\section{Gráfica 3. Subcategorías dentro de la función Ironizar según género}

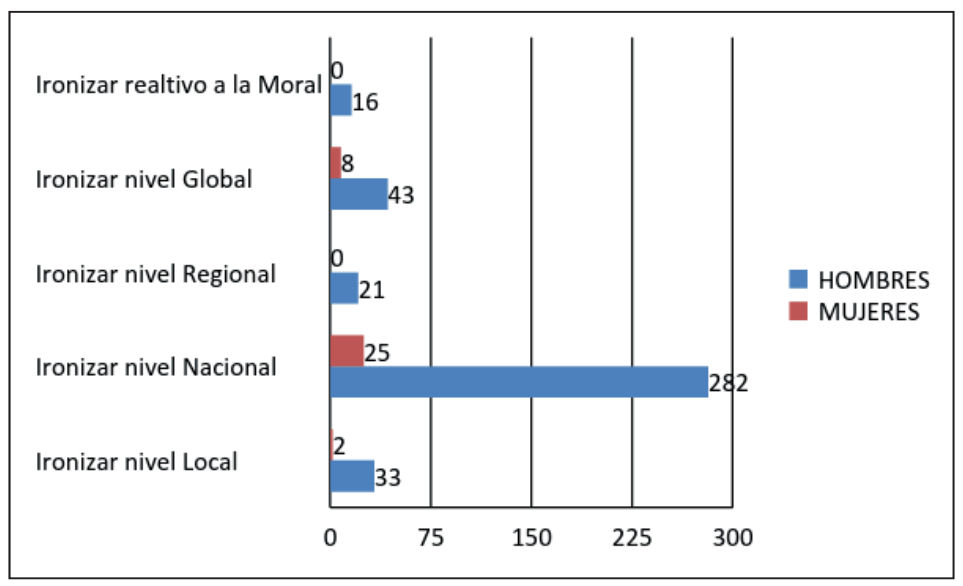

Fuente: elaboración propia.

\subsection{Rasgos en base a las funciones predominantes y perfiles}

En la tabla 4, se pueden observar lo que se ha querido llamar rasgos de los perfiles, que vienen dados por las funciones de mayor producción de tuits de la muestra.

Tabla 4. Rasgos del perfil tomados de las funciones de los tuits

\begin{tabular}{|l|l|}
\hline Funciones de los tuits & Rasgos propuestos para generar perfil \\
\hline Conversar & Conversadores \\
\hline Hacer crónicas del ego & Cronistas del ego \\
\hline Jugar con el lenguaje & Ingeniosos \\
\hline Debatir & Argumentadores \\
\hline Ironizar & Bufones \\
\hline Vigilar, denunciar & Vigiladores \\
\hline Compartir información & Informadores \\
\hline
\end{tabular}

Fuente: elaboración propia.

Finalmente, las combinaciones de estos rasgos detallados en la tabla anterior han sido usadas para ilustrar una especie de perfil. Se podría decir que todos los perfiles de los tuiteros analizados, anónimos, hombres y mujeres, tienen como rasgos predominantes básicos el de Conversadores y Cronistas del ego; sin embargo, vale la pena 
señalar los rasgos que son exclusivos en cada categoría, es decir aparecen solamente en esa tipología de usuario y no en las otras. Por tipología, estos rasgos exclusivos son:

- Tipología mujeres: rasgo Vigiladores (436 tuits)

- Tipología anónimos: rasgo Ingenioso (159 tuits)

- Tipología hombres: rasgo Argumentadores (215 tuits)

Es interesante observar en los tuits de las mujeres una orientación hacia la denuncia, en términos serios, de problemáticas relacionadas a lo público, lo cual desmonta el estereotipo femenino en relación a los intereses. Sin embargo, dado que fue un estudio del caso de una cuenta, no podrían realizarse generalizaciones.

En la gráfica 4 se pueden observar gráficamente la conformación de los perfiles de cada tipología y también se detallan el número de tuits producidos en cada función relacionada al rasgo del perfil.

\section{Gráfica 4. Combinación de funciones con mayor producción total de tuits según tipología de usuario}

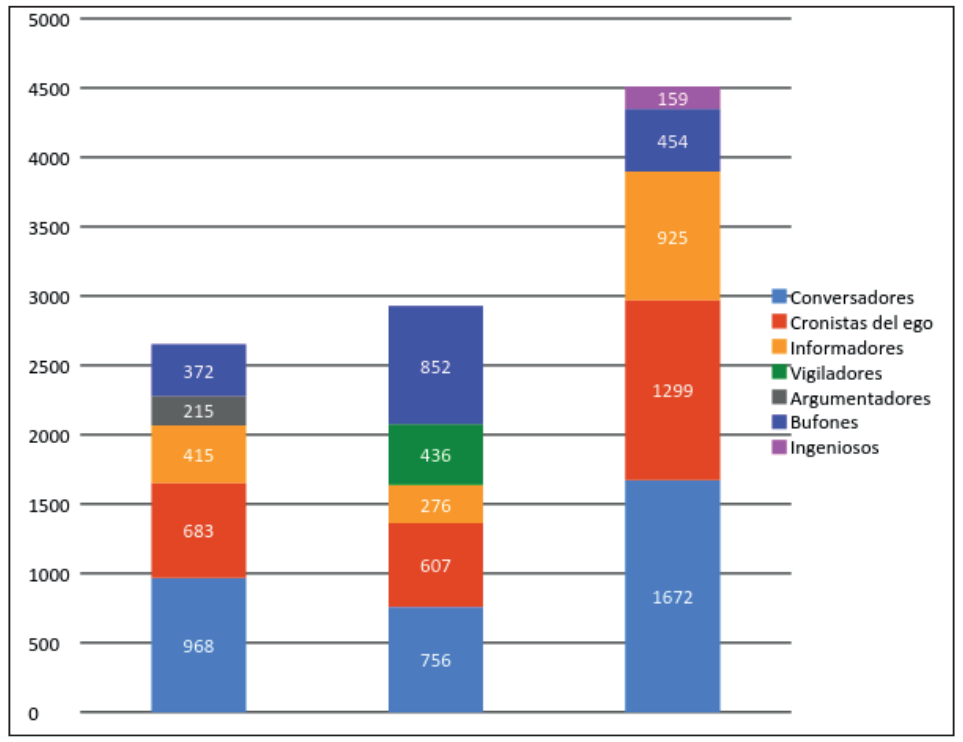

Fuente: elaboración propia.

\section{Conclusiones}

Fue interesante descubrir que al aplicar la metodología de análisis textual, usando categorías basadas en funciones del lenguaje (Jakobson, 1948) y considerando cada tuit, como dirigido a alguien, se pueden evidenciar las interacciones en una red social como Twitter, en la cual se pone claramente de manifiesto las audiencias imaginadas de las que hablan Boyd y Marwick (2011) a las que cada usuario destina sus publicaciones constantemente.

Por otro lado, la ficha diseñada, al incluir el link de cada tuit, podría servir para ilustrar el concepto de aura semántica del hipertexto de Lévy (1999), pues se podría identificar, en base a los tuits que pertenecen a funciones 
como: Hacer crónicas de la propia vida o Auto reconocerse, de qué manera los usuarios van construyéndose a través de estos saltos y referencias hipertextuales.

La categoría No determinado que se incluye en la ficha, podría dar cuenta de funciones emergentes, propias de la dinámica de la red, cuyo contenido valdría la pena explorar más de cerca.

La descripción de las categorías y subcategorías podría perfeccionarse con nuevas pruebas. Esta ficha podría adaptarse también a usuarios de carácter gubernamental o corporativo y podría revelar características interesantes en relación a las funciones que prevalecen en las comunicaciones generales de las cuentas de organismos del Estado, personajes políticos o las interacciones con la ciudadanía.

Después de la prueba realizada y si se aplica a universos de tuits más amplios, la ficha puede ser de utilidad para comprender los procesos intersubjetivos y de interacción de las redes que posibilitaría reflexiones más amplias en relación a la subjetividad contemporánea, la orientación hacia lo público o lo íntimo, la cyberdemocracia, la relación con las nuevas lógicas urbanas. Las funciones de Auto reconocerse y Hacer crónica de la propia vida, podrían ayudarnos a identificar más claramente, en qué medida y de qué manera, los usuarios de Twitter, usan estas estrategias performativas de micro-celebridad (Boyd \& Marwick, 2011) o de yo-pantalla (Sibilia, 2006, 2010) a la hora de administrar su autorepresentación.

\section{Referencias}

1. Abela, J. A. (2002). Las técnicas de análisis de contenido: Una revisión actualizada. Recuperado el 10 de octubre de 2014: http://public.centrodeestudiosandaluces.es/pdfs/S200103.pdf

2. Abreu, C. (2012). La construcción de las identidades virtuales. Recuperado el 12 de octubre de 2013, del sitio web Academia: https://www.academia.edu/3039716/La_construccion_de_las_identidades_digitales._. Identidades_tecnologias_y_subjetividades_en_las_redes_sociales_en_internet

3. Andréu, J. (s.f.). Lastécnicas de Análisis de Contenido: una revisiónactualizada. Recuperado el 10 de diciembre de 2014, del sitio web del Centro de Estudios Andaluces: http://public.centrodeestudiosandaluces.es/ pdfs/S200103.pdf

4. Barbero, J. M. (1998). De los medios a las mediaciones: comunicación, cultura y hegemonía. Bogotá, Colombia: Convenio Andrés Bello.

5. Bardin, L. (1986). Análisis de contenido (3ra. Ed.), Madrid, España: Akal.

6. Bauman, Z. (2000). Modernidad líquida. Buenos Aires, Argentina: Fondo de cultura económica Argentina.

7. Boyd, D. (2011). Dear Voyeur, meet Flâneur... Sincerely, Social Media. Recuperado el 22 de septiembre de 2014, de Library of Queen University: http://library.queensu.ca/ojs/index.php/surveillance-and-society/ article/viewFile/4187/4189

8. Boyd, D. (2008). Taken Out of Context. American Teen Sociality in Networked Publics. Recuperado el 10 de septiembre de 2014, de Danha Boyd website: http://www.danah.org/papers/TakenOutOfContext.pdf

9. Boyd, D. (2010). Tweet, Tweet, Retweet: Conversational Aspects of Retweeting on Twitter. Recuperado el 22 de septiembre de 2014, de HICSS, 2010, 2014 47th Hawaii International Conference on System Sciences: http://www.computer.org/csdl/proceedings/hicss/2010/3869/00/03-06-04-abs.html 
10. Boyd, D., \& Ellison, N. (2007). Social Network Sites: Definition, History, and Scholarship. Recuperado el 18 de septiembre de 2013, de Danah Boyd website: http://www.danah.org/papers/JCMCIntro.pdf

11. Boyd, D., \& Marwick, A. (2011). To See and Be Seen: Celebrity practice on Twitter. Recuperado el 3 de octubre de 2014, de Danh Boyda website: http://www.tiara.org/blog/wp-content/uploads/2011/07/marwick_ boyd_to_see_and_be_seen.pdf

12. Castells, M. (2001). Internet y la sociedad red. Lección inaugural del programa de doctorado sobre la sociedad de la información y el conocimiento. Recuperado el 10 de octubre de 2013, de Engage Intel Website: https://engage.intel.com/servlet/JiveServlet/downloadBody/26111-102-1-31790/INTERNET\%20 Y\%20LA\%20SOCIEDA

13. Costantini, M. (2004). Semiótica de la Comunicación. Revista Razón y palabra, 38.

14. Doucet, A. V. (2008). Análisis de contenido y propuesta de metadatos para la representación documental de la fotografía científica: un estudio de casos. Tesis Doctoral para la obtención del grado de doctor en documentación. Universidad de Granada, Granada, España.

15. Eco, U. (1987). La estrategia de la ilusión. Recuperado el 10 de octubre 2013: http://old.liccom.edu.uy/ bedelia/cursos/semiotica/textos/eco_guerrilla.pdf

16. Fernández, M. Á. B. (2010). Bases teóricas del estructuralismo. Herencia: Estudios literarios, lingüísticos y creaciones artísticas, 2(2), 81-89.

17. Jakobson, R. (1960). Lingüística y Poética. Recuperado el 13 de enero de 2015, del Blog de Uniandes: http:// designblog.uniandes.edu.co/blogs/dise2356/files/2011/03/Jakobson.-Lingu\%CC\%88i\%CC\%81stica-yPoe\%CC\%81tica.pdf

18. Java, A., Song, X., Finin, T., \& Tseng, B. (2007). Why We Twitter: Understanding Microblogging Usage and Communities. Recuperado el 15 de diciembre de 2013, de Website Ebiquity group: http://ebiquity.umbc. edu/_file_directory_/papers/369.pdf

19. Kaiero, A. (2010). Deconstrucción de narrativas y territorios sonoros en los espacios globales abiertos por las redes de comunicación. Recuperado el 10 de septiembre de 2014, del sitio web de Euskomedia: http:// www.euskomedia.org/PDFAnlt/musiker/17/17365388.pdf

20. Lange, M. (2009). From Always on to Always There: Locative Media as Playful Technologies. Recuperado el 4 de octubre de 2014, de BIJT website: http://bijt.org/wordpress/wp-content/uploads/2009/12/ fromalwaysontoalwaysthere_def02.pdf

21. Lévy, P. (1999). ¿Qué es lo virtual? Buenos Aires, Argentina: Paidos.

22. Marwick, A., \& Boyd, D. (2011). I Tweet Honestly, I Tweet Passionately: Twitter Users, Context Collapse, and the Imagined Audience. Recuperado el 26 de septiembre de 2013, de Alice Marwick website: http://www. tiara.org/blog/wp-content/uploads/2010/07/marwick_boyd_twitter_nms.pdf

23. Mischaud, E. (. (2007). Twitter: Expressions of the whole self. An investigation into user appropriation of a web-based communications platform. Recuperado el 2 de diciembre de 2013, de Social Media Centre Stage: http://www.enterprise20.centrestage.de/pdf/Mischaud_final.pdf

24. Nyíri, K. (2006). Time and the Mobile Order. Recuperado el 3 de octubre de 2014, de HUNFI Oktatás website: http://www.hunfi.hu/nyiri/TMO.pdf 
25. Pano, A. (2012). Funciones metalingüísticas y dobles lecturas del código: estudio introductorio a través de los "dardos" de F. Lázaro Carreter, XXIV Congresso AISPI (PAdova, 23-26 maggio 2007), a cura di A. Cassol, A. Guarino, G. Mapelli Bon, P. Taravacci, Roma, AISPI Edizioni, 2012, pp. 607-616.

26. Pew Internet Project. (s.f.). Social Networking Fact Sheet. Recuperado el 12 de octubre de 2014, de Pew Research: http://www.pewinternet.org/fact-sheets/social-networking-fact-sheet/

27. Piñuel J.L. (2002). Epistemología, metodología y técnicas del análisis de contenido. Recuperado el 10 de diciembre 2014, del sitio web de la Universidad Complutense: https://www.ucm.es/data/cont/docs/2682013-07-29-Pinuel_Raigada_AnalisisContenido_2002_EstudiosSociolinguisticaUVigo.pdf

28. Sánchez, M. M. (2000). Los fines del hablar (reflexiones acerca de las funciones del lenguaje, las lenguas y el uso lingüístico). Lynx Documentos de Trabajo, 29.

29. Scolari, C. (2008). HIPERMEDIACIONES. Elementos para una Teoría de la Comunicación digital interactiva. Barcelona, España: Gedisa.

30. Sibilia, P. (2006). La intimidad como espectáculo. Recuperado el 1 de diciembre de 2013, de la Biblioteca virtual: Universidad Javeriana: http://cmap.javeriana.edu.co/servlet/SBReadResourceServlet?rid=1J2SK 927M-22DBXQG-1TB

31. Sibilia, P. S. (2010). La intimidad. Un problema actual del psicoanálisis. Buenos Aires, Argentina: Psicolibro Ediciones.

32. Statistic Brain. (2014). Twitter Company Statistics. Recuperado el 16 de marzo de 2014, de http://www. statisticbrain.com/twitter-statistics/

33. Suarez, B. (2014). ¿Qué estás pensando? El dispositivo de enunciación en la red social Facebook. La Trama de la Comunicación, 18 (83-93) . Recuperado de http://www.redalyc.org/articulo.oa?id=323930547005

34. Vega, P., \& Chirino, C. (2012). Identidad y redes sociales: construcción narrativa. Recuperado el 28 de septiembre de 2014, de Austral Comunicación: http://www.austral.edu.ar/ojs/index.php/ australcomunicacion/article/view/1/5

35. Wesch, M. (2009). YouTube and You: Experiences of Self-awareness in the Context Collapse of the Recording Webcam. Recuperado el 26 de septiembre de 2014, del sitio web de Kansas University: http://krex.k-state. edu/dspace/handle/2097/6302 